\title{
Effects of chirp match and synchronization jitter on gain in optical parametric chirped pulse amplification
}

\author{
Xianhua Li, Shuguang Zeng, Bin Zhang*, \\ Nianchun Sun \\ College of Electronics Information, Sichuan University, \\ Chengdu 610064, Sichuan, China \\ *Corresponding author: zhangbinff@sohu.com
}

\author{
Zhan Sui \\ Laser Fusion Research Center of CAEP, \\ Mianyang 621900, Sichuan, China
}

\begin{abstract}
The method for determining the chirp matched parameter has been proposed by studying the variation of the total gain of OPCPA with the pump optical chirped parameters and the corresponding relationship of signal optical and pump optical wavelengths. Taking nonlinear BBO crystal as a typical example, the effects of chirp match and synchronization jitter on gain in optical parametric chirped pulse amplification have been studied. The results show that the gain spectrum width is greatly broadened by chirp matched method to compensate gain narrowing. For the case of the time synchronization, both sides of the signal optical pulse are amplified efficiently and the gain curve is considerably symmetrical for chirp matched case. While for the case of the relatively large synchronization jitter, the signal optical gain and the gain curve are quite different for different pump optical chirped parameters. It is also concluded that for a given chirp matched degree, with the synchronization jitter increasing, the shifting of gain curve is more obvious and gain curve of signal optical pulse is more asymmetric, and the corresponding gain is lower.
\end{abstract}

Keywords-Chirp match; synchronization jitter; optical parametric chirped pulse amplification; chirped pulse

\section{INTRODUCTION}

In recent years, much interest has been focused on the development of chirped pulse amplification (CPA) acting as a light source for the applications in laser systems such as creating an energetic, ultra-short and ultra-high power pulse. However, in the process of CPA, there are also some shortcomings, including gain narrowing effect, serious thermal effect, decline of pulse signal-to-noise ratio (SNR) after amplification, and easy to produce self-focusing, etc. As a result, the application of CPA technology is subject to certain restrictions [1], [2]. Fortunately, optical parametric chirped pulse amplification (OPCPA) technology based on CPA exhibits high desired gain, wide gain bandwidth, high signalto-noise ratio, minimizing $\mathrm{B}$ integral, low thermal loading effects and other prominent advantages. Consequently, OPCPA is nowadays the most promising technology for producing ultra-short and super-high power laser pulse [3]-[5].

Recently, in order to obtain a wider gain bandwidth, chirp matched OPCPA method has been proposed by controlling the chirped parameter of pump pulse and signal pulse, which arranges the wave vectors of the interacting fields into pairs to meet the phase-matching relations at all times [6]-[8]. However, to our knowledge, there is a lack of literatures about the effects of chirp matched degree on conversion efficiency, gain spectrum, and pulse profile of OPCPA. In addition, in the process of OPCPA, only when the pump and signal pulse are incident upon the nonlinear crystal simultaneously and meet the phase-matching conditions, the signal pulse can be amplified effectively. However, in the process of OPA, there is always time-synchronization jitter between the signal pulse and pump pulse. Thus, a wider pump pulse is generally adopted to interact with a narrower signal pulse to overcome the effects of time-synchronization jitter on the pulse profile, gain, and SNR, etc. Previously, for the case of non-chirped pump pulse, the effects of time-synchronization jitter on the pulse profile and the gain have already been studied [9]. The main aim of this paper is to study the effects of synchronization jitter and chirp matched degree on the gain of OPCPA for the collinear case.

\section{THEORY}

In the plane-wave and the slowly varying envelope approximations, the optical parametric amplification process can be described by the coupled wave equations [10]:

$$
\begin{aligned}
& \frac{\partial E_{\mathrm{s}}}{\partial z}+\frac{n_{s}}{c} \frac{\partial E_{s}}{\partial t}+\alpha_{s} E_{s}=i \frac{\omega_{s} d_{e f f}}{n_{s} c} \frac{1}{\cos ^{2} \beta_{s}} E_{i}^{*} E_{p} \exp (i \Delta k z), \\
& \frac{\partial E_{i}}{\partial z}+\frac{n_{i}}{c} \frac{\partial E_{i}}{\partial t}+\alpha_{i} E_{i}=i \frac{\omega_{i} d_{e f f}}{n_{i} c} \frac{1}{\cos ^{2} \beta_{i}} E_{s}^{*} E_{p} \exp (i \Delta k z), \\
& \frac{\partial E_{p}}{\partial z}+\frac{n_{p}}{c} \frac{\partial E_{p}}{\partial t}+\alpha_{p} E_{p}=i \frac{\omega_{p} d_{e f f}}{n_{p} c} \frac{1}{\cos ^{2} \beta_{p}} E_{s} E_{i} \exp (-i \Delta k z) .
\end{aligned}
$$

In (1)-(3), $E_{j}, \omega_{j}, n_{j}, \alpha_{j}, \beta_{j}(\mathrm{j}$ denotes $\mathrm{p}, \mathrm{s}, \mathrm{i})$ represents the signal, idler and pump optical laser fields, frequency, refractive index, loss factor, Poynting vector walkoff angle for uniaxial crystals, respectively; $c$ is the speed of light in vacuum; $z$ denotes the optical propagation direction; $d_{\text {eff }}$ represents the effective nonlinearity of the amplifying medium; $\Delta k$ is the phase velocity mismatch factor, i.e.,

$$
\Delta k=k_{p}-k_{s} \cos \alpha-k_{i} \cos \beta .
$$


where $\alpha, \beta$ denote the angles between the signal and pump pulse, idle and pump pulse, respectively.

Assuming the signal pulse and pump pulse are the linear chirped pulse, the corresponding electric fields are expressed as:

$$
\begin{aligned}
& E_{s}(t)=E_{s 0} \exp \left[-\frac{1+i C_{s}}{2}\left(\frac{T}{T_{0}}\right)^{2 n}\right], \\
& E_{p}(t)=E_{p 0} \exp \left[-\frac{1}{2}\left(\frac{T-\Delta T}{T_{0 p}}\right)^{2 m}\right] \cdot \exp \left[-\frac{i C_{p}}{2}\left(\frac{T-\Delta T}{T_{0 p}}\right)^{2}\right] .
\end{aligned}
$$

where $T_{0}, T_{0 p}$ denote the 1/e half-width for the signal and pump pulses, respectively; $n$ and $m$ are the super-Gaussian waveform factor; $\Delta T$ is the synchronization jitter between the pump pulse and signal pulse; the linear chirped parameters for the signal and pump pulses are $C_{s}, C_{p}$, respectively.

The total gain of signal optical pulse is given by

$$
G_{\mathrm{tol}}=\frac{U_{s}}{U_{s 0}}
$$

where $U_{s 0}, U_{s}$ represent the signal optical energy before and after OPCPA, respectively.

In the process of OPCPA, with pulse width usually the order of several hundreds ps to ns, signal optical and pump optical group velocity can be considered to be equal and the crystal optical absorption is very small. Consequently, effects of the absorption loss and group velocity mismatch can be neglected. In this paper, the split-step Fourier Transform and fourth-order Runge-Kutta methods are used to numerically simulate the above-mentioned coupled wave equations.

\section{NUMERICAL SimUlation AND ThEORETICAL ANALYSIS}

In the following calculation, a collinear type I phasematching BBO is taken as the nonlinear crystal with a pump at $532 \mathrm{~nm}$ and a signal at $800 \mathrm{~nm}$. The super-Gaussian waveform factors of the signal and pump pulses are $n=1$ and $m=10$, respectively. The pulse width parameters $T_{0}$ and $T_{0 p}$ are $0.72 \mathrm{~ns}$ and $1.5 \mathrm{~ns}$, respectively. The maximum intensity of the initial signal and pump pulses are $3.5 \times 10^{5} \mathrm{~W} / \mathrm{cm}^{2}, 1.0 \times 10^{9} \mathrm{~W} / \mathrm{cm}^{2}$, respectively. The linear chirped parameter for the signal pulse $C_{s}$ is $1.56 \times 10^{4}$. Under these conditions, a $1.06-\mathrm{cm}$-thick BBO crystal is adopted to achieve the optical parametric saturated amplification for the collinear case. The crystal length is 0.6 $\mathrm{cm}$ for small signal amplification.

Fig.1 shows the variation of the pump optical central wavelength with the signal optical central wavelength. As shown in Fig.1, there is an optimum pump wavelength corresponding to a signal wavelength. Consequently, the pump wavelength can be determined according to the signal wavelength to ensure the signal and pump pulses satisfy phasematching requirement every time, resulting in the effective amplification of signal pulse.

Fig.2 displays the variation of the total gain with the pump optical chirped parameters for different crystal lengths. It can be shown in Fig. 2 that with the increase of the pump optical chirped parameters, the total gain increases first and then decreases. The main reason is that the better the chirp match, the smaller the phase mismatch, the higher the total gain. From analysis of Fig.1 and Fig.2, we can conclude that the total gain is the largest gain with pump chirp matched parameter $C_{p m}=3.0 \times 10^{4}$ for the chirp matched case.

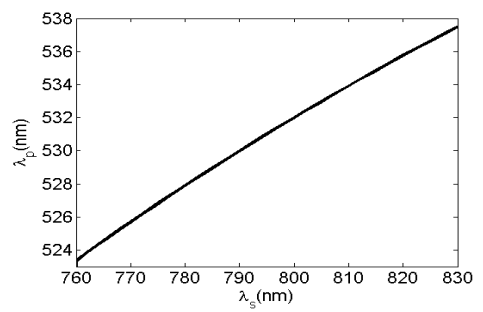

Figure 1. Variation of the pump wavelength with the signal wavelength

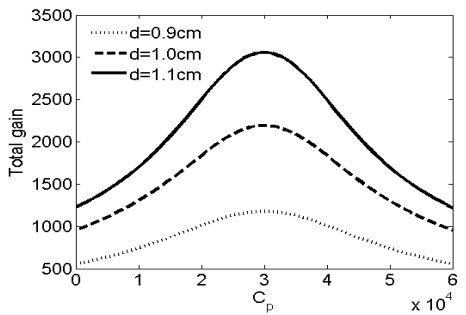

Figure 2. Variation of the total gain with the pump optical chirped parameters

Fig. 3 gives the signal gain spectrum with the different pump optical chirped parameters for the case of the time synchronization, (a) small signal amplification; (b) saturated amplification.
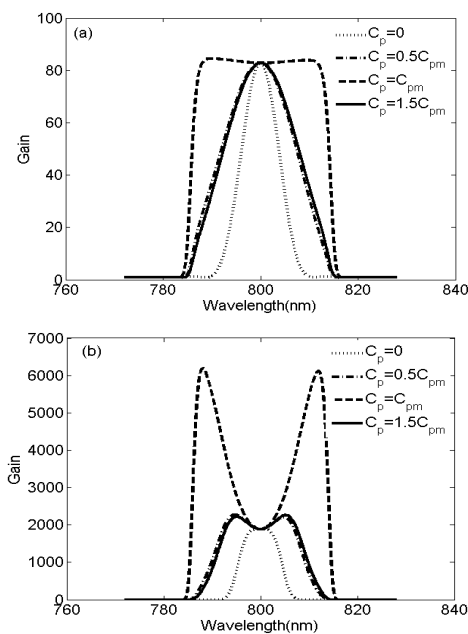

Figure 3. The different pump optical chirped parameters corresponding to the gain spectrum for the synchronization case

Fig. 3 implies that the better the chirp match, the smaller the phase mismatch between signal pulse and pump pulse. Accordingly, gain spectrum bandwidth is broadened to make both sides of the signal pulse be amplified effectively, resulting in compensating gain narrowing. In the process of OPCPA, 
when the crystal length is longer than the best crystals length corresponding to the maximum intensity of signal pulse, the "reverse process" of OPCPA occurs after intensity reaches its maximum. Just then, the energy is from the signal pulse "back" to pump pulse. As a consequent, the middle depression of the signal optical gain appears, while both sides of signal pulse with relatively weak intensity are still amplified effectively.
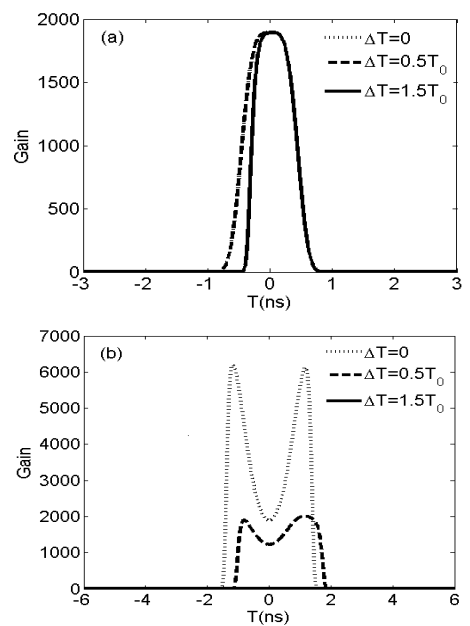

Figure 4. Effects of synchronization jitter on the signal optical gain after optical parametric chirped pulse amplification for saturated amplification case

Fig.4 shows the effects of synchronization jitter on the signal optical gain after OPCPA for the case of saturated amplification, (a) chirp mismatch $\left(C_{p}=0\right)$; (b) chirp match $\left(C_{p}=3.0 \times 10^{4}\right)$. Fig. 4 indicates that the time window of chirp matched signal optical gain is wider than that of chirp mismatched signal optical gain and the middle depression of gain also appears. This is because both sides of the signal pulse meet the phase-matching conditions for the case of chirp match. Thus, gain bandwidth is broadened to ensure gain time window wider. The signal and pump pulses satisfy phasematching requirement every time, resulting in the effective amplification of signal pulse, which is helpful to compensate gain narrowing. In the process of OPCPA, the pulse central signal optical intensity will generate the "reverse process" of OPCPA after intensity reaches its maximum. At the time, the energy will be from the signal pulse "back" to pump pulse. Therefore, the middle depression of the signal optical gain will be extremely apparent. From Fig.4, we can see clearly that for the case of chirp mismatch, when synchronization jitter reaches a certain value, the asymmetric phenomenon of the leading and trailing edge of gain appears significantly. While for the case of chirp match, with synchronization jitter increasing, the drifting of gain curve is more and more serious and asymmetric phenomenon is more apparent, and gain becomes smaller and smaller. The main reason is that if the pump pulse and signal pulse can not enter the nonlinear crystal simultaneously, the signal pulse will not be amplified uniformly, and the leading edge and trailing edge of signal pulse will also be amplified unevenly, resulting in apparent asymmetric phenomenon. For the case of chirp match, with synchronization jitter increasing, the signal optical amplification will be seriously inconsistent with the pump pulse. The signal pulse within the range of the pump optical amplification can still be amplified, whereas the signal pulse without the range of the pump optical amplification is unable to be amplified effectively, resulting in the serious asymmetry and smaller gain.

Fig. 5 shows the effects of chirp match on the signal optical gain after OPCPA for the case of saturated amplification, (a) synchronization $(\Delta T=0)$; (b) non-synchronization $\left(\Delta T=T_{0}\right)$. It can be seen from Fig.5 that for the case of the time synchronization, the chirp match is better and better, the signal amplification exhibits better and better effect on compensating gain narrowing, but the middle of signal optical gain spectrum is sinking. For the case of the comparatively large synchronization jitter, with the chirp match increasing, drifting and distortion will be more serious, and the gain is lower. The main reason is that for the case of the time synchronization jitter, with chirp match increasing, both sides of the signal and pump pulses can interact more effectively to meet the phasematching conditions so that both sides of the signal pulse can achieve an effective amplification and the gain narrowing can also be compensated. The middle signal optical gain generates the "reverse process" of OPCPA, resulting in depression. For the case of the comparatively large synchronization jitter, the better the chirp match, the better the effective amplification of both sides of the signal pulse. However, the amplified range of pump pulse will move obviously, resulting in the signal optical amplification uneven. As a result, a serious distortion phenomenon and lower gain occur.
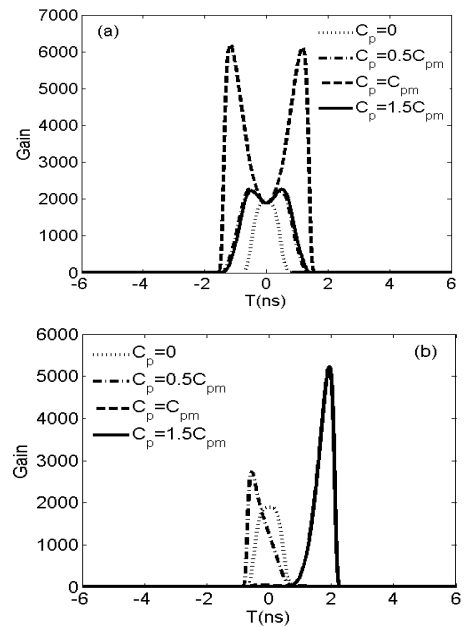

Figure 5. Effects of chirp match on the signal optical gain after optical parametric chirped pulse amplification for saturated amplification case

Fig.6 gives the variation of the total gain with the synchronization jitter for different pump optical chirped parameters for the case of saturated amplification. As shown in Fig. 6, for a given chirped parameter, with the synchronization jitter increasing, the total gain becomes smaller and smaller. The chief reason is that for the case of a given chirped parameters, with the synchronization jitter increasing, the signal pulse out of the range of the pump optical amplification can not be amplified effectively, and the total gain decreases. While for the case of time synchronization, the signal pulse is within the range of the pump optical amplification, resulting the sufficient amplification. For the case of a given 
synchronization jitter, the total gain is apparently different for different chirp matched degree, as can be seen from Fig. 6. The main reason is that for the case of a given synchronization jitter, with different chirp matched degree, the degree of phase mismatch is different, so that the amplification is also different.

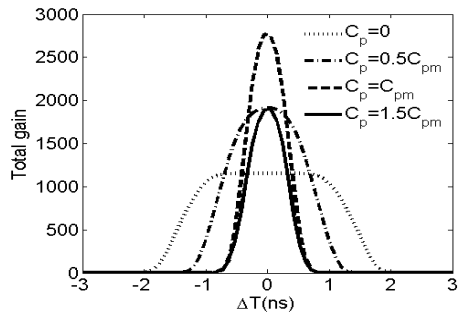

Figure 6. For the case of saturated amplification, variation of the total gain with the synchronization jitter for different pump optical chirped parameters

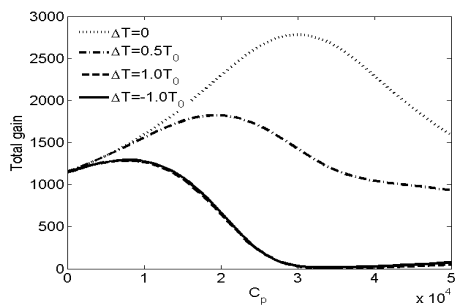

Figure 7. For the case of saturated amplification, variation of the total gain with pump optical chirped parameters for different synchronization jitter

Fig. 7 gives the variation of the total gain with pump optical chirped parameters for different the synchronization jitter for the case of saturated amplification. It can be shown in Fig. 7 that for a given synchronization jitter, with the pump optical chirped parameters increasing, the peak total gain occurs. The main reason is that for the case of a given synchronization jitter, the degree of phase-matching between the signal pulse and pump pulse changes apparently, and the signal pulse and pump pulse can not meet the original phase-matching relations every time, so that each peak total gain corresponds to a different pump optical chirp parameter. Fig.7 also shows that for the case of a given pump optical chirped parameter, the larger the synchronization jitter, the smaller the total gain. The main reason is that for a given pump optical chirped parameter, with synchronization jitter increasing, part of the signal pulse is out of the range of the pump optical amplification, and can not be amplified effectively.

\section{CONCLUSION}

In summary, we demonstrate and study effects of degree of chirp match and synchronization jitter on gain curve and total gain in OPCPA. The results show that the gain narrowing can be compensated effectively in the process of the OPCPA by the method for chirp match of signal and pump optical pulses. Chirp match and synchronization jitter between the signal pulse and pump pulse have a significant influence on gain and total gain. For a given chirp matched degree, with the increase synchronization jitter, noticeable drifting and asymmetric phenomenon of the gain curve appear, and the total gain is also lower. For the case of the time synchronization, both sides of the signal pulse with weak intensity are amplified very well and the gain curve is considerably symmetrical when the parametric amplification is well chirp matched. While for the case of the relatively large synchronization jitter, signal optical gain and gain curve after amplification are quite different for different pump optical chirp parameters, and each peak total gain is corresponded to a different pump optical chirp parameter. Thus, for the case of the time synchronization, due to narrow gain bandwidth for BBO collinear case, high signal optical gain can be obtained by chirp matched method in OPCPA. While parametric fluorescence is out of gain bandwidth so that it can not be amplified effectively to achieve the purpose of enhancing signal-to-noise ratio, which can provide a new method for improving the signal-to-noise ratio for OPCPA. In addition, for the case of the chirp match, the increasing pump optical pulse width can overcome the effects of synchronization jitter on OPCPA, so that the signal pulse can be effectively amplified at all times. Consequently, the signal pulse can be amplified effectively by controlling chirp match and synchronization jitter, and the noise can also be restrained, which can provide a valuable reference for designing of high energy and peak power laser systems.

\section{ACKNOWLEDGMENT}

This work was supported by the National Hi-Tech. Foundation of China.

\section{REFERENCES}

[1] N. Ross, P. Matousek, M. Towrie, A. J. Langley and J. L. Collier. "The prospects for ultrashort pulse duration and ultrahigh intensity using optical parametric chirped pulse amplifiers," Opt.Commun, vol.144, no.23, pp.125-133, Dec.1997

[2] S. Backus, C. G. Durfee III, M. M. Murnane and H. C. Kapteyn. "High power ultrafast lasers," Review of Scientific Instruments, vol.69, no.3, pp.1207-1222, Mar.1998

[3] A. Dubietis, G. Jonuauskas, A. Piskarskas. "Powerful femtosecond pulse generation by chirped and stretched pulse parametric amplification in BBO crystal," Opt.Commun, vol.88, no. 4-6, pp.437440, 1992

[4] J. Moses, C. Manzoni, et al. "Temporal optimization of ultrabroadband high-energy OPCPA," Opt.Express, vol.17, no.7, pp.5540-5555, Mar.2009

[5] N. Ross and P. Matoused. "Analysis and optimization of optical parametric chirped pulse amplification," Opt.Soc.Am, vol.19, no.12, pp.2945-2956, Dec.2002

[6] K. Osvay, I. N. Ross. "Broadband Sum-frequency gennration by chirpassisted group-velocity matching," Opt.Soc.Am, vol.13, no.7, pp.14311438, Jul.1996

[7] J. Limpert, C. Aguergaray, S. Montant, et al. " Ultra-broad bandwidth parametric amplification at degeneracy," Opt.Express, vol.13, no.19, pp.7386-7392, Sep.2005

[8] Y. Tang, I. N. Ross, C. Hernandez-Gomez, et al. "Optical parametric chirped-pulse amplification source suitable for seeding high-energy systems," Opt.Lett, vol.33, no.20, pp.2386-2388, Oct.2008

[9] X. M. Zeng, X. F. Wei, et al. "Effects of synchronization-time jitter in optical parametric chirped-pulse amplification on gain stability," High Power Laser and Particle Beams, vol.18, no.4, pp.529-532, Apr.2006

[10] R.A. Baumgartner, R.L. Byer. "Optical parametric amplification," IEEE J. Quantum Electron, vol.15, no.6, pp.432-444, Jun.1979 